\title{
ANÁLISIS BIBLIOMÉTRICO DE LA PRODUCCIÓN CIENTÍFICA SOBRE VIH/SIDA EN EL PERÚ 1985 - 2010
}

\author{
Patricia Caballero ${ }^{1,2, a}$, César Gutiérrez ${ }^{2, b}$, Gustavo Roselli,c, Martín Yagui 1,b, Jorge Alarcón²,b, \\ Manuel Espinoza ${ }^{1,2, a}$, Cristina Magan ${ }^{3, d}$, José Luis Sebastián ${ }^{3, c}$, César Cabezas ${ }^{2,4, a}$, Franco Romaní ${ }^{2, c}$
}

\begin{abstract}
RESUMEN
Objetivos. Analizar la evolución de la publicación de artículos de investigación sobre VIH/SIDA en Perú. Métodos. Estudio bibliométrico de los artículos sobre VIH/SIDA publicados en revistas indizadas en Medline, SciELO y LILACS hasta octubre de 2010. Se seleccionaron investigaciones desarrolladas completamente en Perú, o estudios multicéntricos con participación de sedes peruanas. Resultados. Se identificó 257 artículos sobre VIH/SIDA, observando un incremento desde el 2003. El promedio de demora de publicación fue de 2,8 $\pm 1,8$ años. Solo $94(36,6 \%)$ artículos fueron publicados en español. Las áreas más estudiadas fueron epidemiología (36,6 \%) y clínica $(35,8 \%)$. El diseño transversal fue el más frecuente $(56,8 \%)$, seguido por las series de casos. Según la clasificación de OMS predominaron los estudios destinados a conocer más la enfermedad y los factores de riesgo (85,6 \%) y según las áreas de intervención el 46,7 \% se enfocó en el diagnóstico y tratamiento. La mayoría de estudios se realizaron en Lima $(65,9 \%)$. El 48,2 \% de estudios se enfocaron en las personas afectadas por el VIH/SIDA. Finalmente, la Revista Peruana de Medicina Experimental y Salud Pública fue la que publicó más artículos sobre VIH/SIDA $(9,7 \%)$. Conclusiones. Se evidencia un crecimiento en la producción científica sobre VIH/SIDA en el Perú, sin embargo, consideramos que las investigaciones no se han basado en una agenda nacional consensuada y basada en prioridades de investigación, lo que ha podido limitar su diseminación y aplicación.
\end{abstract}

Palabras clave: VIH, Síndrome de Inmunodeficiencia Adquirida; Bibliometría; Publicaciones científicas y técnicas, Portales de acceso a revistas científicas; Perú (fuente: DeCS BIREME).

\section{BIBLIOMETRIC ANALYSIS OF SCIENTIFIC PRODUCTION ABOUT HIV/AIDS IN PERU 1985 - 2010}

\begin{abstract}
Objective. To analyze the evolution of published scientific articles on HIVIAIDS in Peru. Methods. A bibliometric analysis of papers on HIVIAIDS published in journals indexed in MEDLINE, SciELO and LILACS until October 2010. We selected research papers fully developed in Peru, and multicenter studies with participating Peruvian sites. Results. We identified 257 publications on HIVIAIDS, showing an increase since 2003. The average publication delay was $2.8 \pm 1.8$ years. Only $94(36.6 \%)$ articles were published in Spanish. The most studied areas were epidemiology $(36.6 \%)$ and clinical topics (35.8\%). The cross-sectional design was the most frequent $(56.8 \%)$ followed by case series. According to the WHO classification, studies to learn more about the disease and risk factors predominated $(85.6 \%)$ and according to the intervention areas, $46.7 \%$ focused on diagnosis and treatment. Most studies were conducted in Lima (65.9\%). $48.2 \%$ of studies focused on people living with HIVIAIDS. Finally, Revista Peruana de Medicina Experimental y Salud Pública was the journal that published most articles on HIVIAIDS (9.7\%). Conclusions. We found a growth in scientific production on HIV / AIDS in Peru; however, we believe that the research undertaken was not based on an agreed national agenda or national research priorities, which might have limitted its dissemination and application.
\end{abstract}

Keywords: HIV; Acquired Immunodeficiency Syndrome; Bibliometrics; Scientific and technical publications; Portals for scientific journals; Peru (source: MeSH NLM).

\section{INTRODUCCIÓN}

En Perú el primer caso de SIDA fue notificado en 1983 en Lima y, desde entonces, se ha desarrollado una epidemia "concentrada", con una prevalencia en población general debajo del $1 \%$, que es mayor al $5 \%$ en poblaciones vulnerables ${ }^{(1)}$. Según el Ministerio de Salud (MINSA), hasta el año 2009 fueron notificados 39890 casos de VIH y 25636 de SIDA. En el mismo año, la razón de masculinidad para SIDA fue 2,7, un valor menor al encontrado al inicio de la epidemia, como consecuencia del incremento relativo de caso en mujeres por la

Oficina General de Investigación y Transferencia Tecnológica. Instituto Nacional de Salud. Lima, Perú.

Instituto de Medicina Tropical “Daniel A. Carrión”, Universidad Nacional Mayor de San Marcos. Lima, Perú.

Estrategia Sanitaria Nacional de Prevención y Control de ITS y VIH-SIDA, Ministerio de Salud. Lima, Perú.

Instituto Nacional de Salud. Lima, Perú

Médico Infectólogo; ${ }^{b}$ Médico, epidemiólogo; c Médico salubrista; ${ }^{d}$ Enfermera

Recibido: 12-05-11 Aprobado: 24-08-11 
transmisión heterosexual hacia la población general (2). La respuesta nacional al VIH ha evolucionado, desde una larga fase inicial de fortalecimiento de instituciones, gestión de recursos y elaboración de guías nacionales para el control y prevención del VIH e infecciones de transmisión sexual, hasta la implementación del programa gratuito de tratamiento antirretroviral de gran actividad (TARGA) desde 2002, y la aprobación del primer Plan Estratégico Nacional Multisectorial para los años 2007 al 2011, que cuenta con la participación y compromiso de instituciones públicas y privadas ${ }^{(3)}$. Sin embargo, esta respuesta no se ha acompañado de pautas rectoras del desarrollo de investigaciones científicas, de tal manera que se genere evidencia suficiente y pertinente sobre las diferentes áreas de intervención en este problema de salud pública.

A nivel internacional, se ha observado un crecimiento sostenido de la investigación sobre VIH/SIDA, relacionado, según algunos autores, al aumento en el número de individuos afectados ${ }^{(4)}$. Como factores relacionados a una mayor productividad de estudios sobre VIH/SIDA se encuentran un mayor número de casos, mayor número de revistas indizadas y la inversión del gasto en salud ${ }^{(5)}$. Una estrategia de análisis de la producción científica la constituyen los estudios bibliométricos, que buscan caracterizar cuantitativamente el desarrollo histórico de las publicaciones, patrones de autoría, uso de dichas publicaciones, entre otros temas ${ }^{(6,7)}$. Según un estudio, el año 2003 los países con mayor número de publicaciones médicas sobre VIH/SIDA fueron países desarrollados (Estados Unidos de Norteamérica, Reino Unido, Francia, Italia y Alemania) (4). A nivel de los países en desarrollo la producción científica sobre VIH/SIDA ha sido analizada en algunos estudios bibliométricos usando metodologías distintas, como en Haití (8), Cuba ${ }^{(9)}$, Nigeria ${ }^{(10)}$, India ${ }^{\left({ }^{11}\right)}$ y el África subsahariana ${ }^{(5,12)}$.

Durante el año 2009 el MINSA, a través del Instituto Nacional de Salud (INS), promovió la identificación de las prioridades nacionales y regionales de investigación en salud para el periodo 2010-2014. Como resultado de este proceso participativo, en nueve de los veinte talleres regionales, se incluyó como prioridad de investigación al VIH/SIDA y, en el foro nacional, se determinó que las investigaciones sobre enfermedades transmisibles (investigaciones operativas y evaluaciones del impacto de las intervenciones actuales y nuevas) debían ser incluidas en el grupo de las siete prioridades de investigación para el Perú ${ }^{(13)}$. Dentro de estas investigaciones prioritarias en enfermedades transmisibles están incluidas las investigaciones en VIH/SIDA, para lo cual se planificó la elaboración de una agenda nacional de investigación. En este contexto, el INS y la Estrategia Sanitaria Nacional de Prevención y Control de ITS-VIH/SIDA, consideraron necesario realizar un estudio bibliométrico de las investigaciones realizadas en el país, previo a la elaboración de la agenda nacional de investigación, con la finalidad de orientar las decisiones y los recursos adecuadamente.

El objetivo del presente estudio fue explorar el desarroIlo de las publicaciones de investigaciones sobre $\mathrm{VIH} /$ SIDA realizadas en Perú, lo que ayudará a conocer las fortalezas, capacidades y limitaciones de la investigación sobre VIH/SIDA en el Perú.

\section{MATERIALES Y MÉTODOS}

Los artículos fueron obtenidos a través de una búsqueda documentaria realizada en octubre de 2010 en las bases Medline ${ }^{(14)}$, SciELO (Perú y regional) ${ }^{(15)}$ y LILACS ${ }^{(16)}$. Elegimos dichas bases por su cobertura y la rigurosidad científica que se exige para la inclusión de revistas. Se seleccionaron los artículos de investigaciones desarrolladas completamente en Perú, o estudios multicéntricos con participación de sedes peruanas, independientemente de la nacionalidad de los investigadores. No se incluyeron informes técnicos, libros o resúmenes de congresos. Los términos de búsqueda fueron: HIV and Peru, Acquired Immunodeficiency Syndrome and Peru, AIDS and Peru, Sexual transmitted disease and Peru, STD and Perú, SIDA y Perú, Síndrome de Inmunodeficiencia humana y Perú, ITS y Perú e Infecciones de transmisión sexual y Perú. No se hizo ninguna restricción temporal.

Se elaboró una base de datos en el programa Excel con las variables consignadas en el siguiente párrafo. El registro de información fue realizado por dos investigadores con experiencia en publicación de artículos científicos, teniendo un control de calidad por un tercer investigador. Solo se incluyeron artículos originales, comunicaciones cortas y reportes de casos, no se consignaron notas, cartas, editoriales ni artículos de revisión.

Para cada artículo se registraron las siguientes variables: título del estudio, año de publicación, tiempo de demora de publicación en años, idioma, área de investigación, diseño del estudio (17), tipo de investigación de la OPS (18) (investigación para conocer la enfermedad y factores de riesgo, evaluaciones de impacto de intervenciones actuales, evaluaciones de impacto de intervenciones nuevas, evaluación costo-efectividad, investigación operativa, investigaciones para sustentar inversiones en investigación en salud y para medir el impacto y costo beneficio de las investigaciones en la salud), áreas temáticas de intervención por el MINSA, lugar donde se realizó el estudio, población de estudio, instituciones participantes y factor de impacto de la revista científica ${ }^{(12)}$ 


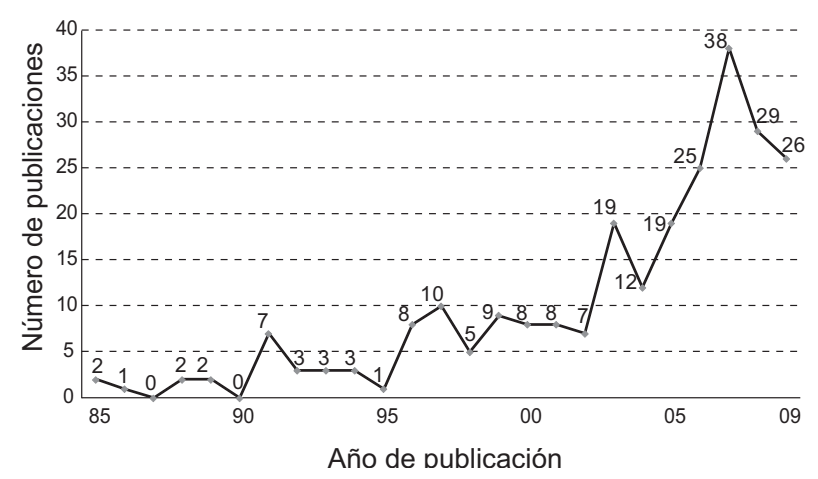

Figura 1. Número de artículos científicos sobre VIH/SIDA en el Perú, 1985-2009.

empleándose el Journal Citation Reports (JCR) del año 2009 (http://scientific,thomson,com/products/jcr/).

Se realizó un análisis descriptivo (frecuencias y porcentajes) de cada una de las variables usando el programa Excel MS 2007 para Windows.

\section{RESULTADOS}

Se encontraron 257 artículos sobre VIH/SIDA, publicados desde 1985 hasta octubre de 2010. A partir del año 2003 ocurre un incremento importante en el número de artículos sobre este tema, publicándose 178 desde ese año hasta octubre 2010 (69,8 \% de los artículos identificados). La Figura 1 presenta la evolución del número de artículos por año hasta 2009 (para esta figura, no se considera el año 2010 porque solo se registraron publicaciones hasta el mes de octubre).
En 110 (42,8 \%) artículos se pudo determinar el tiempo transcurrido la finalización del estudio y su publicación. El promedio de demora de publicación fue 2,8 \pm 1,8 años. El 4,5\% de artículos se publicó el mismo año en que terminó el estudio, el 20,9\% al año siguiente, el $23,6 \%$ dos años después y otro $23,6 \%$ a los tres años. El tiempo máximo de demora de publicación fue nueve años. El $63 \%$ de artículos fue publicado en inglés, 36,6\% en español, y solo uno $(0,4 \%)$ en portugués. El 36,6 \% de artículos correspondió al estudio de la epidemiología del VIH/SIDA y el 35,8 \% a aspectos clínicos. En menor frecuencia los artículos corresponden a salud pública e intervenciones (19,1\%), ciencias básicas (5,4\%), gestión de servicios (1,9\%) y economía de la salud (1,2\%). Los artículos de economía de la salud recién son identificados en el quinquenio 2000-2004, mientras que aquellos sobre gestión de servicios en el quinquenio 2005-2010, como se aprecia en la Figura 2.

El diseño más frecuente fue el transversal (56,8 \%), el 9,3\% fue reporte/serie de caso, el 7,8 \% estudios de cohortes o longitudinales, el 7,0 \% revisiones sistemáticas (que trataron principalmente sobre la prevalencia de la infección por $\mathrm{VIH}$, así como la frecuencia de conductas de riesgo, pero sin llegar a realizar un metanálisis) y el 6,3\% a ensayos clínicos. Con menor frecuencia los estudios correspondieron a otros diseños. Según la clasificación de la OPS, el 85,6 \% de artículos fueron investigaciones para conocer más la enfermedad y los factores de riesgo, el 11,7\% estudios sobre evaluaciones de impacto de intervenciones actuales, 1,6 \% evaluaciones de costo-efectividad de intervenciones y $1,1 \%$ investigaciones operativas.

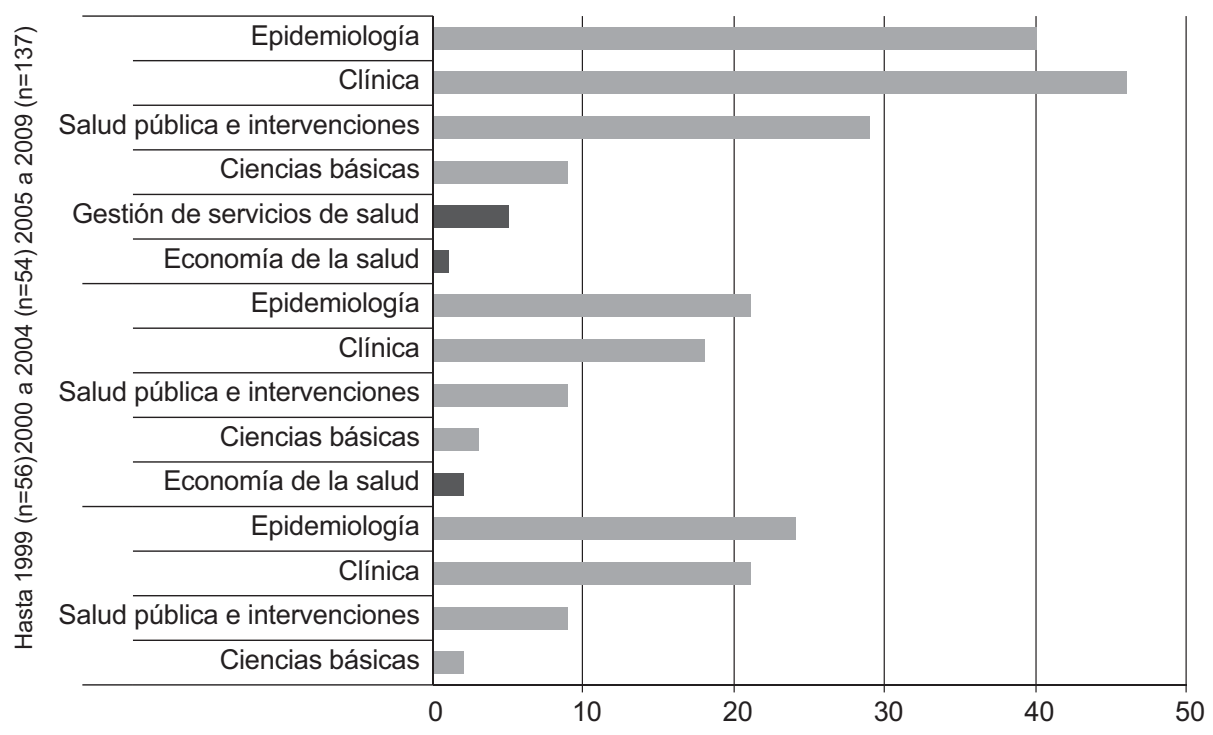

Figura 2. Área de investigación de los artículos científicos sobre VIH/SIDA en el Perú según periodos, 1985-2009. 
Respecto a las áreas temáticas de intervención del MINSA el 46,7 \% se enfocó en el diagnóstico y tratamiento del $\mathrm{VIH} / \mathrm{SIDA}, 12,8 \%$ fue sobre diagnóstico y tratamiento de ITS y $10,5 \%$ sobre atención preventiva en poblaciones de alto riesgo y vulnerabilidad. Con menor frecuencia, los artículos trataron sobre atención integral de personas que viven con VIH $(7,8 \%)$, medidas de prevención en adolescentes (4,7\%), estrategias de consejería y tamizaje $(4,3 \%)$, educación para la prevención (4,3\%), gestión de los servicios (3,9\%), atención de gestantes serorreactivas y niños expuestos al VIH $(3,1 \%)$, atención preventiva en poblaciones indígenas y afrodescendientes $(1,6 \%)$ y atención de gestantes serorreactivas y niños expuestos a la sífilis $(0,4 \%)$.

En 255 artículos se pudo determinar el lugar de ejecución del estudio. La mayoría se realizó solo en Lima $(65,9 \%)$. El $18,8 \%$ fue realizado en Lima y provincias, el $8,6 \%$ solo en provincias y el $6,7 \%$ se trataron de estudios donde Perú era una sede de investigación de estudios internacionales multicéntricos. Respecto al grupo estudiado, 124 artículos $(48,2 \%)$ se enfocaron en la población de personas que viven con VIH/SIDA (PVVS) y 51 (19,8\%) en grupos de la población general. El detalle de los grupos estudiados se muestra en la Figura 3.

En 256 artículos se pudo identificar a las instituciones participantes del artículo. En 139 (54,3 \%) hubo participación internacional, de estas, en 52 artículos la participación fue únicamente de instituciones internacionales sin asociación con otras peruanas. Dentro de los 117 $(45,7 \%)$ artículos sin participación internacional, las publicaciones realizadas por instituciones privadas peruanas fueron las más frecuentes, como se ve en la Figura 4. La participación de instituciones extranjeras en publicaciones sobre VIH/SIDA ha ido aumentando en el tiempo; en 1999, el 45,5\% de las publicaciones tuvieron participación extranjera; el periodo 2000-2004, la participación extranjera fue del 50,9 \% y en el periodo 2005-2009 fue del 59,1\%. Las instituciones peruana y extranjera con mayor participación en los artículos sobre

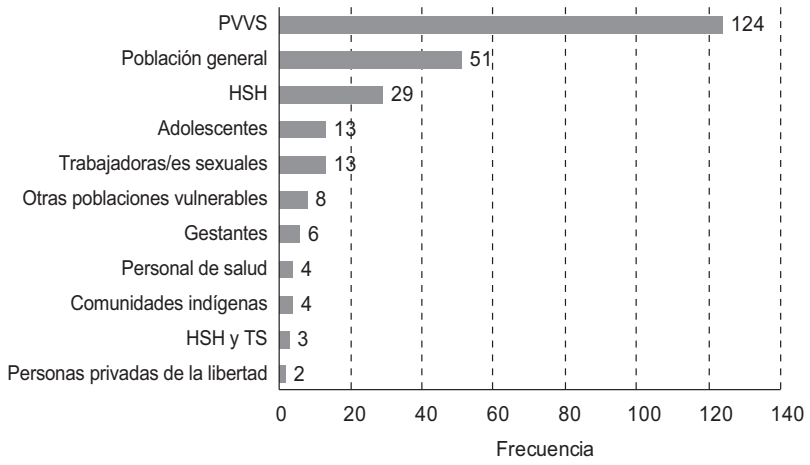

Figura 3. Grupo de estudio en los artículos científicos sobre VIH/SIDA en el Perú.

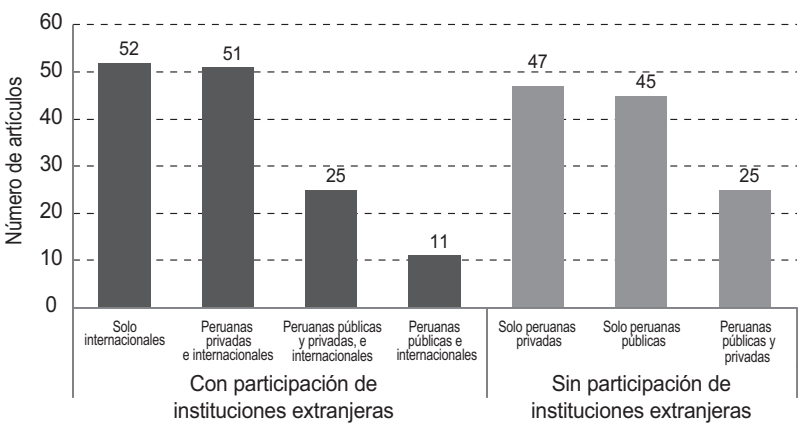

Figura 4. Tipo de instituciones que participan en los artículos sobre VIH/SIDA en el Perú.

VIH/SIDA son la Universidad Peruana Cayetano Heredia con 105 publicaciones y la Universidad de Washington con 36 publicaciones, respectivamente. La Tabla 1 muestra las diez primeras instituciones nacionales e internacionales con publicaciones sobre VIH/SIDA en Perú.

Los artículos analizados fueron publicados en 78 revistas biomédicas, el 33,1 \% en revistas nacionales y el 66,9\% en internacionales. La Tabla 2 muestra las primeras diez revistas donde se publicaron más artículos, las que concentran el 53,3 \% de las publicaciones, el resto se distribuye entre 68 revistas médicas diferentes. Basado en la búsqueda de los factores de impacto de las revistas

Tabla 1. Instituciones nacionales e internacionales que han publicado artículos científicos sobre VIH/SIDA en el Perú.

\begin{tabular}{|c|c|}
\hline Institución & $\begin{array}{l}\text { Número } \\
\text { de publi- } \\
\text { caciones }\end{array}$ \\
\hline \multicolumn{2}{|l|}{ Nacionales } \\
\hline 1. Universidad Peruana Cayetano Heredia & 105 \\
\hline 2. Instituto Nacional de Salud & 21 \\
\hline 3. Universidad Nacional Mayor de San Marcos & 20 \\
\hline 4. Hospital Nacional Cayetano Heredia & 17 \\
\hline 5. Asociación Civil Impacta Salud y Educación & 17 \\
\hline 6. Asociación Benéfica PRISMA & 13 \\
\hline 7. Hospital Nacional Dos de Mayo & 7 \\
\hline 8. Hospital Nacional Guillermo Almenara Irigoyen & 7 \\
\hline 9. Dirección General de Epidemiologia & 6 \\
\hline 10. Ministerio de Salud & 6 \\
\hline \multicolumn{2}{|l|}{ Internacionales } \\
\hline 1. Universidad de Washington & 36 \\
\hline $\begin{array}{l}\text { 2. Unidad de Investigación de la Marina de los } \\
\text { Estados Unidos }\end{array}$ & 26 \\
\hline 3. Universidad de California & 22 \\
\hline 4. Escuela de Medicina de Harvard & 21 \\
\hline 5. Universidad Johns Hopkins & 14 \\
\hline $\begin{array}{l}\text { 6. Centro de control y prevención de } \\
\text { enfermedades (CDC) }\end{array}$ & 7 \\
\hline 7. National Health Institute (NIH) & 7 \\
\hline $\begin{array}{l}\text { 8. Departamento de Salud Pública de San } \\
\text { Francisco }\end{array}$ & 7 \\
\hline 9. Imperial College London & 6 \\
\hline 10. Fred Hutchinson Cancer Research Center & 5 \\
\hline
\end{tabular}


Tabla 2. Principales revistas médicas donde se publicaron los artículos científicos sobre VIH/SIDA en Perú.

\begin{tabular}{lccc}
\hline \multicolumn{1}{c}{ Revista } & n & \% & $\begin{array}{c}\text { Factor de } \\
\text { impacto }\end{array}$ \\
\hline $\begin{array}{l}\text { Revista Peruana Medicina } \\
\text { Experimental y Salud Pública }\end{array}$ & 25 & 9,7 & N.D \\
Revista Médica Herediana & 21 & 8,2 & N.D \\
$\begin{array}{l}\text { Journal of Acquired Immune } \\
\text { Deficiency Syndrome }\end{array}$ & 20 & 7,8 & 4,57 \\
AlDS & 14 & 5,4 & 4,90 \\
Sexually Transmitted Diseases & 12 & 4,7 & 2,57 \\
$\begin{array}{l}\text { Revista de Gastroenterología del } \\
\text { Perú }\end{array}$ & 11 & 4,3 & N.D \\
Anales Facultad de Medicina & 10 & 3,9 & N.D \\
$\begin{array}{l}\text { American Journal of Tropical } \\
\text { Medicine and Hygiene }\end{array}$ & 9 & 3,5 & 2,79 \\
Journal of Infectious Diseases & 8 & 3,1 & 5,86 \\
$\begin{array}{l}\text { AlDS Research and Human } \\
\text { Retroviruses }\end{array}$ & 7 & 2,7 & 2,17 \\
\hline
\end{tabular}

N.D: no disponible

biomédicas en el año 2009, 146 (56,8 \%) de los artículos fueron publicados en revistas que tienen registrado un factor de impacto. Solo cinco artículos fueron publicados en revistas con un factor de impacto de diez o más (The Lancet, Science y Annals of Internal Medicine), siendo mayor la publicación en revistas con un factor de impacto entre dos y menos de tres $(20,6 \%)$ o entre cuatro y menos de cinco $(17,9 \%)$.

\section{DISCUSIÓN}

Este artículo es un análisis descriptivo acerca de las publicaciones sobre VIH/SIDA realizados en el Perú durante más de dos décadas. Desde la publicación de los primeros artículos en 1985 han pasado 25 años durante los cuales se ha observado un incremento sostenido en la producción literaria de VIH/SIDA del Perú. El primer caso de SIDA fue diagnosticado en el Perú en 1983 y

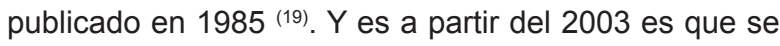
da un marcado incremento en la producción científica sobre VIH/SIDA, publicándose desde entonces 178 artículos en menos de siete años; este incremento se observa también en otros estudios ${ }^{(8-10)}$. Sin embargo, en producción total, el número de publicaciones sobre VIH/SIDA en el Perú es mucho menor a la descrita en la India, Sudáfrica, Zimbabue, Zambia y Haití en periodos más cortos ${ }^{(8,11,12)}$, o comparada a la producción en revistas de enfermería brasileñas, donde se encontró 55 publicaciones sobre VIH/SIDA entre 1983 y $1999{ }^{(20)}$.

Aunque solo se pude determinar el tiempo de demora en publicación en el $48 \%$ de los artículos analizados, que no necesariamente reflejan el verdadero tiempo editorial; la mayoría de publicaciones fueros hechas entre uno a tres años luego de finalizado el estudio, esta variable no ha sido evaluada en otros estudios similares.
Respecto al área de investigación el 36,6 \% de artículos fueron sobre epidemiologia, 35,8 \% fueron sobre clínica y $19,1 \%$ fueron sobre salud pública. Otros estudios han tratado de describir el área de investigación con clasificaciones diversas, en Nigeria el 29,5 \% de artículos fueron sobre salud pública, ocupacional y ambiental, $14,2 \%$ sobre inmunología y $10,6 \%$ virología ${ }^{(10)}$. En un estudio en países del sur de África los temas de mayor cobertura fueron educación para la salud, estudios en infantes y estudios de conducta sexual ${ }^{(12)}$. En Haití, los temas de investigación son distintos, casi el $40 \%$ de los artículos tratan sobre etnología, en especial en haitianos nacidos de personas con VIH/SIDA y que viven en Canadá o los Estados Unidos, el $60 \%$ restante de artículos corresponden a epidemiologia ${ }^{(8)}$. En Cuba la temática es terapia $(24,6 \%)$, epidemiología $(21,1)$, prevención y control $(21,1 \%)$, mortalidad $(8,3 \%)$, complicaciones $(5,2 \%)$, etiología $(4,1 \%)$, economía $(3,8 \%)$ y diagnóstico $(2,4 \%)^{(9)}$. En Brasil, en el estudio de revistas de enfermería, el 30,7 \% fue sobre cuidado asistencial, $22 \%$ prácticas educativas, 17,3\% transmisión vertical, $16,7 \%$ experiencia de vida y $10 \%$ aspectos epidemiológicos ${ }^{(19)}$. La cobertura temática presenta entonces variaciones entre países, no todos los países le otorgan la misma prioridad a los problemas que el VIH/SIDA plantea, sin embargo, un tema recurrente es la epidemiología.

Además, describimos la cobertura de las áreas de investigación por periodos, observando que los estudios epidemiológicos y clínicos son los más publicados en todos los periodos y que los estudios sobre salud pública e intervenciones se incrementan progresivamente hasta ubicarse en el tercer lugar a partir del año 2005, pasando de cuatro publicaciones antes del año 2005 hasta 22 publicaciones luego a este periodo. Es importante resaltar que a partir del año 2000 los estudios de economía de la salud empiezan a publicarse, así como los estudios sobre gestión de servicios a partir del año 2005. La variable diseño de investigación tampoco ha sido descrita por otros estudios bibliométricos sobre VIH/SIDA. Nuestros resultados indican que los estudios transversales son los más frecuentes $(56,8 \%)$, seguido por los reporte/serie de casos (9,3\%). Los artículos han abordado como tema de investigación más frecuentes el diagnóstico y tratamiento del VIH/SIDA, seguido del diagnóstico y tratamiento de ITS y la atención preventiva en poblaciones de alto riesgo o vulnerables; esta caracterización no ha sido realizada en estudios bibliométricos previos sobre VIH/SIDA.

Desde el año 2004 en adelante se desarrolló el 61 \% de las publicaciones sobre diagnóstico y tratamiento de VIH/SIDA, mostrando una clara relación con el inicio del programa de acceso gratuito al tratamiento antirretroviral (TARGA) y los proyectos de intervención financiados por el Fondo Mundial. La misma tendencia se observa con la publicación de los estudios sobre diagnóstico y 
tratamiento de ITS. De manera comparativa, los estudios realizados en población de gestantes y niños expuestos al VIH o la sífilis, solo son el $3,1 \%$ y $0,4 \%$, respectivamente.

Respecto a los ensayos clínicos, se han encontrado 16 publicaciones, 13 de estudios randomizados y tres de no randomizados. Sin embargo, a través de información registrada en el Instituto Nacional de Salud, entidad reguladora nacional, encontramos que se ha autorizado la ejecución en el país de 51 ensayos clínicos sobre VIH/SIDA solamente entre 1998 y 2010 , de los cuales 38 (75\%) fueron autorizados desde 2005, posterior a la implementación del programa de acceso gratuito al TARGA. Sería necesario conocer los resultados de todos los estudios autorizados y además analizar las razones de la falta de publicación.

Respecto a la participación de instituciones, a diferencia de India ${ }^{(11)}$, Sudáfrica, Zimbabue y Zambia ${ }^{(12)}$ donde la mayoría de publicaciones han sido realizadas solo por instituciones nacionales, en nuestro país el 54,2 \% de artículos ha tenido participación de alguna institución internacional, similar a Haití ${ }^{(6)}$ y Nigeria ${ }^{(8,10)}$. En todos los casos el país con mayor colaboración es Estados Unidos de Norteamérica, seguido por el Reino Unido. La participación internacional en el Perú ha ido en aumento, de $45,5 \%$ en el periodo 1985-1999 hasta 59,1\% en el periodo 2005-2009. Dentro de las instituciones internacionales las Universidades de Washington, California, Harvard y Johns Hopkins son las que más han publicado sobre VIH/SIDA en el Perú, junto con la Unidad de Investigación de la Marina de los Estados Unidos (NAMRU-6). El perfil de las instituciones es similar al encontrado por un estudio que caracterizó las redes de colaboración institucional en la producción científica peruanas en revistas indizadas en el Institute for Scientific Información (ISI). En dicho estudio son las instituciones extranjeras las que tienen una mayor producción, siendo la mayoría de ellas universidades. En el Perú, solo dos universidades (Universidad Peruana Cayetano Heredia y Universidad Nacional Mayor de San Marcos) son las principales productoras, habiendo una mayor diversidad de instituciones no universitarias como institutos públicos especializados de salud, hospitales y organismos no gubernamentales que investigan y publican ${ }^{(22)}$.

Si bien la mayoría de artículos (67 \%) fueron publicados en revistas internacionales, dentro de las primeras diez revistas donde más se ha publicado artículos sobre VIH/ SIDA tenemos cuatro nacionales: Revista Peruana de Medicina Experimental y Salud Pública, Revista Médica Herediana, Revista de Gastroenterología del Perú y Anales de la Facultad de Medicina. Además, solo el $56,8 \%$ de artículos estuvieron publicados en revistas con factor de impacto disponible en el año 2009 , porcentaje menor al encontrado en el estudio bibliométrico en Nigeria (81,9\%). El 7,4\% de los artículos estuvieron publicados en revistas con factor de impacto de entre cinco y menos de diez, mientras que 1,9\% fueron publicados en revistas con factor de impacto de diez o más. Dicho porcentaje es similar al encontrado en Nigeria ${ }^{(8)}$, donde $6,7 \%$ de artículos fueron publicados en revistas con factor de impacto igual o mayor de cinco.

Como potenciales limitaciones del estudio están relacionadas a las bases de datos utilizadas, las cuales no representan toda la literatura biomédica y científica publicada, existiendo otras bases de datos importantes como ISI, SCOPUS, entre otras. Sin embargo, consideramos que las bases seleccionadas logran la recuperación de la mayor cantidad de publicaciones sobre $\mathrm{VIH} / \mathrm{SIDA}$ realizada en nuestro país. Otras limitaciones son la no inclusión del país colaborador o número de autores por publicación. También debe considerarse el sesgo de publicación, ya que las bases examinadas no tienen registradas toda la investigación sobre $\mathrm{VIH} /$ SIDA realizada en el Perú, mucha de la cual no llega a publicarse. La utilización del factor de impacto para valorar la revista médica puede traer problemas para la comparación de resultados, ya que puede cambiar cada año y las metodologías de los estudios previos para el registro del factor de impacto es distinta. Los pocos estudios bibliométricos sobre este tema y las distintas metodologías usadas, sobre todo en las bases usadas, hacen menos válidas las comparaciones realizadas, pero al no ser el objetivo del estudio, dichas comparaciones brindan un panorama de la realidad en nuestro medio de este tipo de investigación. Por último, algunos autores que trabajan en instituciones públicas desde donde extraen los datos para sus investigaciones aparecen como investigadores de algunas universidades privadas e incluso internacionales lo que hace difícil su categorización, y podría estar sobreestimada la participación internacional en las publicaciones sobre VIH/SIDA en el Perú.

A pesar que la producción científica sobre VIH/SIDA se ha incrementado en los últimos años, el volumen de producción en el Perú es bajo en comparación con países desarrollados y otros en vías de desarrollo. La investigación que se hace es básicamente de tipo epidemiológica y clínica y el diseño transversal es el más usado. La mayoría de artículos tuvo participación de instituciones extranjeras y cerca del 70 \% se publicó en revistas extranjeras. El desarrollo de publicaciones sobre epidemiología, clínica y salud pública e intervenciones se han incrementado coincidiendo con el mismo periodo en que los programas dirigidos por el Ministerio de Salud han sido fortalecidos y ampliados en el ámbito nacional.

Durante el periodo estudiado, las investigaciones realizadas no estuvieron basadas en una agenda nacional 
de investigación consensuada y basada en prioridades, lo que ha podido limitar la diseminación y la aplicación de sus resultados dentro de las políticas públicas. Es por ello necesario contar con una agenda nacional de investigación en VIH/SIDA en la que participen todos los actores involucrados en los temas como investigadores, gestores y decisores de políticas públicas, desde el sector público y el privado.

\section{AGRADECIMIENTOS}

Agradecemos el apoyo de ONUSIDA en el Perú, en especial Renate Ehmer y Patricia Bracamonte, por el asesoramiento e información brindada.

\section{Contribuciones de autoría}

PC, CG, GR y MY participaron en la concepción y diseño del trabajo, CG y FR en la recolección de información, PC, CG, GR, MY, JA, ME y FR en el análisis e interpretación de datos, PC, CG y FR en la redacción del manuscrito, GR, MY, JA, ME, CM, JLS y CC en la revisión crítica del manuscrito, $\mathrm{PC}, \mathrm{MY}, \mathrm{ME}$ y $\mathrm{CC}$ en la obtención de financiamiento. Todos los autores aprobaron la versión final del trabajo.

\section{Fuentes de financiamiento}

Presupuesto del Instituto Nacional de Salud

\section{Conflictos de interés}

No existe conflicto de interés de los autores.

\section{REFERENCIAS BIBLIOGRÁFICAS}

1. Dirección General de Epidemiología. Análisis de la situación epidemiológica del HIVISIDA en el Perú, Bases Epidemiológicas para la prevención y control. Lima: Ministerio de Salud del Perú; 2006.

2. Dirección General de Epidemiología. Análisis de la situación de salud del Perú, 2010. Lima: Ministerio de Salud; 2006.

3. Cáceres C, Mendoza W. The National Response to the HIV/ AIDS Epidemic in Peru: Accomplishments and Gaps - A Review. J Acquir Immune Defic Syndr. 2009;51:S60-6.

4. Ramos J, Gutierrez F, Padilla S, Masia M, Martin-Hidalgo A. Geography of medical publications. An overview of HIV/ AIDS research in 2003. AIDS. 2005;19(2):219-20.

5. Uthman O. Pattern and determinants of HIV research productivity in sub-Saharan Africa: bibliometric analysis of 1981 to 2009 PubMed papers. BMC Infect Dis. 2010;10:47.

6. Young $\mathbf{H}$. The ALA glossary of library and information science. Chicago: American Library Association; 1983.

7. Rehn C, Kronman U. Bibliometric handbook for Karolinska Institutet. Huddinge: Karolinska Institutet; 2008.
8. Macias C. AIDS in Haiti: a bibliometric analysis. Bull Med Libr Assoc. 2000;88(1):56-61.

9. Santovenia J. Análisis bibliométrico realizado al Bibliomed dedicado al Síndrome de Inmunodeficiencia Adquirida. Revista Cubana de Informática Médica. 2006;6(2).

10. Uthman O. HIVIAIDS in Nigeria: a bibliometric analysis. BMC Infect Dis. 2008;8:19.

11. Kumar S, Chand P. HIVIAIDS research in India: A bibliometric study. Library \& Information Science Research. 2007;29:124-34.

12. Macías C, Mijangos A, Eguía J, Ramírez M, García G. Análisis bibliométrico de la literatura publicada sobre sida en el sur de África. Rev Esp Doc Cient. 2002;25(4):433-41.

13. Caballero $P$, Yagui $M$, Espinoza M, Castilla $T$, Granados A, Velazquez A, et al. Prioridades regionales y nacionales de investigación en salud, Perú 2010-2014: Un proceso con enfoque participativo y descentralista. Rev Peru Med Exp Salud Publica. 2010;27(3):398-411.

14. U.S. National Library of Medicine, PubMed.gov [Internet]. Bethesda, MD: NCBI; c2009 [citado el 2 agosto de 2011]. Disponible en: http://www.ncbi.nlm.nih.gov/pubmed/

15. Scientific Electronic Library Online [Internet]. São Paulo: SciELO; [citado el 2 agosto de 2011]. Disponible en: http:// www.scielo.org/php/index.php?lang=es

16. Biblioteca virtual en Salud [Internet]. São Paulo: Bireme/ OPS/OMS; [citado el 2 agosto de 2011]. Disponible en: http:// regional.bvsalud.org/php/index.php?lang=es

17. Rothman K, Greenland S. Modern Epidemiology. 2da Ed. Filadelfia: Lippincott Williams \& Wilkins; 1998.

18. Instituto Nacional de Salud. Guía del facilitador. Proceso de definición de prioridades nacionales de investigación en salud. Lima: Ministerio de salud; 2009.

19. Peralta T, Pichilingue P, Ruiz W, Chahud A, Alcántara J, Patrucco R. Sindrome de inmunodeficiencia adquirida: Reporte de un caso. Rev Gastroenterol Peru. 1985;5:139-49.

20. Reis R, Gir E. Caracterização da produção científica sobre doenças sexualmente transmissíveis e HIVIAIDS publicados em periódicos de enfermagem do Brasil. Rev Esc Enferm USP. 2002;36(4):376-85.

21. Costa R, Padilha M, Vieira M, Alves I, Bastiani J. La contribución de la Enfermería brasileña para la producción de conocimiento sobre el SIDA. Index Enferm. 2009;18(1):70-4.

22. Huamani C, Mayta P. Producción científica peruana en medicina y redes de colaboración, análisis del Science Citation Index 2000-2009. Rev Peru Med Exp Salud Publica. 2010;27(3):315-25.

Correspondencia: Patricia Caballero Ñopo

Dirección: Jr. Capac Yupanqui N. ${ }^{\circ}$ 1400; Jesús María. Lima, Perú.

Teléfono: (+511) 617-6200 anexo 2175

Correo electrónico: pcaballe@ins.gob.pe 